\title{
Long-term dynamic changes of NMDA receptors following an excitotoxic challenge
}

\author{
Alberto Granzotto ${ }^{1,2,3, *}$, Marco d'Aurora ${ }^{1}$, Manuela Bomba ${ }^{1,2}$, Valentina Gatta ${ }^{1,4}$, Marco Onofrj ${ }^{2}$, \\ Stefano L. Sensi ${ }^{1,2,5}$
}

${ }^{1}$ Center for Advanced Sciences and Technology (CAST), University “G. d'Annunzio" of Chieti-Pescara, Chieti, Italy

2 Department of Neuroscience, Imaging, and Clinical Sciences (DNISC), Laboratory of Molecular Neurology, University “G. d'Annunzio" of Chieti-Pescara, Chieti, Italy

${ }^{3}$ Sue and Bill Gross Stem Cell Research Center, University of California - Irvine, Irvine, USA

${ }^{4}$ Department of Psychological, Health and Territorial Sciences (DISPUTer), Laboratory of Molecular Genetics, University "G. d'Annunzio" of Chieti-Pescara, Chieti, Italy

${ }^{5}$ Center of Advanced Studies and Technology, University “G. d'Annunzio" of Chieti-Pescara, Chieti, Italy

* To whom correspondence should be addressed:

Alberto Granzotto

Center for Advanced Sciences and Technology (CAST)

University G. d'Annunzio of Chieti-Pescara,

Via Colle dell'Ara, Chieti 66100, Italy.

e-mail: alberto.granzotto@unich.it 


\section{Abstract}

Excitotoxicity is a form of neuronal death characterized by the sustained activation of N-methyl-Daspartate receptors (NMDARs) triggered by the excitatory neurotransmitter glutamate. NADPHdiaphorase neurons [also known as nNOS (+) neurons] are a subpopulation of aspiny interneurons, largely spared following excitotoxic challenges. Unlike nNOS (-) cells, nNOS (+) neurons fail to generate reactive oxygen species in response to NMDAR activation, a key divergent step in the excitotoxic cascade. However, additional mechanisms underlying the reduced vulnerability of nNOS (+) neurons to NMDAR-driven neuronal death have not been explored. Using functional, genetic, and molecular analysis in striatal cultures, we demonstrate that $\mathrm{nNOS}(+)$ neurons possess distinct NMDAR properties. These specific features are primarily driven by the peculiar redox milieu of this subpopulation. In addition, we found that nNOS (+) neurons exposed to a pharmacological maneuver set to mimic chronic excitotoxicity alter their responses to NMDAR-mediated challenges. These findings suggest the presence of mechanisms providing long-term dynamic regulation of NMDARs that can have critical implications in neurotoxic settings.

Keywords: Calcium, Reactive Oxygen Species, Nitric Oxide Synthase, NADPH diaphorase, Neurodegeneration, Excitotoxicity 


\section{Introduction}

$\mathrm{N}$-methyl-D-aspartate receptors (NMDARs) are ionotropic glutamatergic receptors primarily permeable to calcium ions $\left(\mathrm{Ca}^{2+}\right)$. Transient NMDAR-driven $\mathrm{Ca}^{2+}$ influx mediates essential physiological or pathological functions (Choi, 1988, 2020; Paoletti et al., 2013). In pathological conditions, NMDAR overstimulation generates a $\mathrm{Ca}^{2+}$-dependent cascade of events encompassing the production of reactive oxygen and nitrogen species (ROS and RNS, respectively), irreversible mitochondrial failure, and zinc $\left(\mathrm{Zn}^{2+}\right)$ mobilization, ultimately leading to neuronal demise (Choi, 2020; Sensi et al., 2009; Wang and Swanson, 2020). The process, termed excitotoxicity (Choi, 1992, 2020; Lau and Tymianski, 2010), is critical for the development of acute or chronic neurological conditions, like stroke, traumatic brain injury, Alzheimer's disease, Huntington's disease, and Parkinson's disease (TBI, AD, HD, and PD, respectively) (Bano et al., 2011; Beal, 1998; Choi, 2020; Hynd et al., 2004).

NADPH-diaphorase neurons [also known as nNOS (+) neurons] are a subpopulation of medium-sized aspiny interneurons, spared following excitotoxic challenges (Granzotto and Sensi, 2015; Koh et al., 1986; Koh and Choi, 1988; Uemura et al., 1990; Weiss et al., 1994). This subpopulation is characterized by the naïve overexpression of the neuronal form of the enzyme nitric oxide synthase (NOS, also known as NOS1) (Dawson et al., 1991; Hope et al., 1991). Notably, early studies have demonstrated nNOS (+) neurons are spared in post-mortem brain samples obtained from AD, HD, and PD patients (Ferrante et al., 1985; Graveland et al., 1985; Mufson and Brandabur, 1994), thereby indicating selective resilience to neurodegeneration.

In two recent studies, we have exploited the distinct features of these neurons to dissect molecular mechanisms associated with the resistance to excitotoxic hits (Canzoniero et al., 2013; Granzotto and Sensi, 2015). These studies have indicated that, in response to NMDA exposures, nNOS (+) neurons produce intracellular $\mathrm{Ca}^{2+}$ rises $\left(\left[\mathrm{Ca}^{2+}\right]_{\mathrm{i}}\right)$ that largely overlap with those observed in the general population of nNOS (-) neurons. However, nNOS (+) neurons fail to generate ROS of mitochondrial origin, mobilize neurotoxic amount of $\mathrm{Zn}^{2+}$ from intracellular pools and undergo mitochondrial damage (Granzotto et al., 2020).

This study explored distinct subtle differences in NMDAR composition/distribution in nNOS $(+)$ neurons and investigated whether these features offer additional neuroprotective effects. Two lines of evidence support this working hypothesis. First, NMDARs exert different activities according to their subunit composition and synaptic localization (Hardingham and Bading, 2010; Paoletti et al., 2013). Second, it is unclear how nNOS (+) neurons cope with the late-stage (also termed amplification stage) of excitotoxicity, a phase in which damaged neurons spread the toxic cascade to 
neighboring cells (Zivin and Choi, 1991). Also, not completely clear is the behavior of these cells upon chronic neurodegenerative conditions like AD and HD (Lewerenz and Maher, 2015).

\section{Materials and methods}

\section{Chemicals}

Culture media and sera were purchased from GIBCO (Thermo Fisher Scientific). Fluorescent indicators (fluo-4 AM, fura-2 AM, fura-FF AM) were purchased from Molecular Probes (Thermo Fisher Scientific). NMDA was from Merck Millipore. MK-801 and NBQX were from Alomone. All the other chemicals, unless otherwise stated, were from Sigma-Aldrich.

\section{Neuronal striatal cultures}

All the procedures involving animals were approved by the institutional Ethics Committee (47/2011/CEISA/COM) and carried out following national and international laws and policies. Female mice were caged in groups while male mice were singly housed. Mice were kept on a 12:12 light/dark cycle and had ad libitum access to food and water. All efforts were made to minimize animal suffering during procedures.

Neuronal striatal cultures were prepared as previously described (Granzotto and Sensi, 2015). Briefly, after tissue collection and enzymatic/mechanical dissociation, single-cell striatal suspension was diluted in Neurobasal medium supplemented with $0.5 \mathrm{mM}$ L-glutamine, $5 \%$ horse serum, $5 \%$ fetal bovine serum, $1 \times$ B 27 and $0.2 \%$ penicillin/streptomycin and plated onto pre-treated laminin/poly-DL-lysine coated tissue culture plates or dishes. To prevent non-neuronal cell growth and to obtain near-pure striatal cultures, three days after plating, the medium was supplemented with $5 \mu \mathrm{M}$ of cytosine arabinofuranoside (Ara-C). The $7^{\text {th }}$ day in vitro (DIV) $25 \%$ of the medium was replaced with fresh Neurobasal. Experiments were performed on cultures between 12 and 18 DIV.

\section{NADPH-diaphorase staining}

NADPH-diaphorase staining was employed for ex-post identification of nNOS (+) neurons, as previously described (Canzoniero et al., 2013; Granzotto and Sensi, 2015). Briefly, after microfluorimetry experiments, cells were washed with ice-cold Tris-buffer (TBS), fixed in 4\% paraformaldehyde (PFA)/0.1 M phosphate buffer (PBS) for up to 30 minutes at $4^{\circ} \mathrm{C}$, rinsed with large volumes of TBS, and incubated for $30-60$ minutes at $37^{\circ} \mathrm{C}$ with freshly made NADPH- 
diaphorase staining solution containing (in $\mathrm{mM}$ ): $100 \mathrm{Tris} / \mathrm{HCl}, 1.2$ sodium azide, 0.2 nitrotetrazolium blue, 1 NADPH (Merck-Millipore), and 0.2\% Triton X-100, pH 7.2.

\section{Sample collection for mRNA analysis}

Sample collection for mRNA analysis of $\mathrm{nNOS}(+)$ and $\mathrm{nNOS}(-)$ neurons was performed as described elsewhere (Kim et al., 2001) with some modifications. Striatal cultures were rinsed in icecold TBS buffer and fixed in 4\% PFA for 15 minutes at $4^{\circ} \mathrm{C}$. After PFA removal, cells were thoroughly washed in TBS, permeabilized with Tris $/ \mathrm{HCl}+0.2 \%$ Triton X100, and stained with the NADPHdiaphorase staining method. The staining solution was then removed, and cells were treated for $20-$ $60 \mathrm{~s}$ with Proteinase $\mathrm{K}(1 \mu \mathrm{g} / \mathrm{ml})$ in TE buffer (Invitrogen - Thermo Fisher Scientific). Single nNOS (+) neurons were identified on the stage of an upright microscope, aspirated with a patch pipette, and transferred into a $1.5 \mathrm{ml}$ conical tube containing mRNA lysis buffer (PicoPure RNA Isolation Kit Thermo Fisher Scientific). Similarly, nNOS (-) neurons of similar size and shape were harvested as controls. 11 to 18 neurons per sample were collected from 3 independent cultures. Neurons were lysed and processed following manufacturer instructions and total RNA stored at $-80^{\circ} \mathrm{C}$ until further analysis. All these procedures were performed by employing DEPC water and DNAse/RNAse-free chemicals.

$q R T-P C R$ analysis

The PicoPure RNA isolation kit (Thermo Fisher Scientific) was employed for total RNA extraction. One $\mu \mathrm{g}$ of RNA was retro-transcribed with the High-Capacity RNA-to-cDNA Kit (Thermo Fisher Scientific). qRT-PCR was carried out on an Abi 7900HT Sequencing Detection System (Thermo Fisher Scientific) in a total volume of $25 \mu$ l containing: 2x Maxima SYBR Green/ROX qPCR Master Mix (Thermo Fisher Scientific), $1 \mu \mathrm{L}$ of cDNA and $0.3 \mu \mathrm{M}$ of each primer. Gapdh and Hprt1 were employed as endogenous controls. Amplification conditions were as follow: 2 minutes at $50^{\circ} \mathrm{C}$, 10 minutes at $95^{\circ} \mathrm{C}$, followed by 40 cycles of 15 seconds at $95^{\circ} \mathrm{C}$ and 1 minute at $60{ }^{\circ} \mathrm{C}$. A melting curve was run to assess the specificity of primers employed. Samples were run in triplicate. The gene relative fold changes were calculated by the $\Delta \Delta \mathrm{Ct}$ method. The employed primers were: Gapdh (Forward 5'-AACAGCAACTCCCACTCTTC-3', Reverse 5'-GTGGTCCAGGGTTTCTTACTC-3'), Gpx1 (F 5'CGACATCGAACCTGACATAGA-3', R 5'-CAGAGTGCAGCCAGTAATCA-3'), Grin1 (F 5'GTGAACGTGTGGAGGAAGAA-3', R 5'-GTGGAGGTGATAGCCCTAAATG-3'), Grin2b (F 5'GTCCCTTTATCCTCCGTCTTTC-3', R 5'-CGTCGACTCTCTTGGTTTGTAG-3'), Grin2a (F 5'GCTACTGGAGGGCAACTTATAC-3', R 5'-TGGTCTGGCAAGAGAGATTTG-3'), Hprt1 (F 5'GGCCAGACTTTGTTGGATTTG-3', R 5'-CGCTCATCTTAGGCTTTGTATTTG-3'), Nos1 (F 5'- 
CTCGGTCTTTGTCTCTCTTTCTT-3', R 5'-GGATGTGATGTGGTAGGGTTAG-3'), Sod2 (F 5'GTAGAGCCTTGCCTGTCTTATG-3', R 5'-AAACCCAGAGGCACCATTAC-3').

\section{Bioinformatic analysis}

Whole cortex and hippocampus scRNA-Seq data were obtained from the SMART-Seq Allen Brain Atlas database (portal.brain-map.org) and retrieved on March 26 $6^{\text {th }}, 2020$ (Allen Brain Institute, 2019; Lein et al., 2007). nNOS (+) neurons were identified by filtering for neuronal cells showing a high expression of Nos1 transcripts (>10.0 FPKM) and the abundant expression of additional nNOS(+) neuron markers (Gad, Sst, Pvalb, and Npy). Two cell clusters (Sst Chodl_1, Sst Chodl_2) were identified (Supplementary File 1). Differentially expressed gene (DEG) analysis was performed using Cytosplore Viewer, a publicly available visual analysis system to interrogate single-cell data published in the Allen Cell Types Database (Tasic et al., 2018). To limit the number of differentially expressed genes and given the heterogeneity of the neuronal subtypes, the nNOS (+)neuron transcriptome was compared with the transcriptome of GABAergic neurons (Supplementary File 2).

\section{Live-cell imaging}

All live-cell imaging experiments were performed on an epifluorescent Zeiss Axio Examiner.D1 upright microscope equipped with a Xenon lamp-based Cairn Optoscan monochromator, a Zeiss 20x NA 1.0 W Plan-Apochromat water immersion objective, and selective fluorescence emission filters. Images were acquired with a Photometrics 16-bit Evolve 512 EMCCD camera and analyzed with the Molecular Devices Metafluor 7.7 software.

\section{$\mathrm{Ca}^{2+}{ }_{i}$ imaging experiments}

Striatal cultures were loaded for $30 \mathrm{~min}$ in the dark at room temperature (RT) with fluo-4 AM $(3 \mu \mathrm{M})$, fura-2 AM (3 $\mu \mathrm{M})$, or fura-FF AM $(5 \mu \mathrm{M})$ plus $0.1 \%$ Pluronic F-127 in a HEPES-controlled saline solution (HCSS) containing (in $\mathrm{mM}$ ): $120 \mathrm{NaCl}, 5.4 \mathrm{KCl}, 0.8 \mathrm{MgCl}_{2}, 1.8 \mathrm{CaCl}_{2}, 20 \mathrm{HEPES}, 15$ glucose, $10 \mathrm{NaOH}$, and pH 7.4. Cells were then washed and incubated in the dark for further $30 \mathrm{~min}$ in HCSS. fluo-4 (excitation $\lambda$ : $473 \pm 20 \mathrm{~nm}$, emission $\lambda$ : $525 \pm 25 \mathrm{~nm}$ ) fluorescence changes of each cell were expressed as $\Delta F / F$, where $F$ is the fluorescence intensity at rest and $\Delta F$ the relative fluorescence change $\left(F_{X}-F\right)$ over time. Similarly, fura-2 and fura-FF (excitation $\lambda: 340 \pm 10 \mathrm{~nm}, 380 \pm$ $10 \mathrm{~nm}$, emission $\lambda: 510 \pm 45 \mathrm{~nm}$ ) fluorescence changes of each cell were acquired as 340/380 emission ratio and expressed as $\Delta R / R$, where $R$ is the fluorescence ratio at rest, and $\Delta R$ is the relative fluorescence ratio change $\left(\mathrm{R}_{\mathrm{x}}-\mathrm{R}\right)$ over time (Csernansky et al., 1994). During all $\left[\mathrm{Ca}^{2+}\right]_{i}$ 
measurements, TPEN (200 - 500 nM, Merck Millipore) was added to the bathing solution to prevent interferences of the fluorescent dyes with heavy metal ions (i.e., $\mathrm{Zn}^{2+}$ ) (Grynkiewicz et al., 1985).

\section{Analysis of spontaneous $\mathrm{Ca}^{2+}{ }_{i}$ transients}

Spontaneous $\mathrm{Ca}^{2+}{ }_{i}$ changes were acquired at a $5 \mathrm{~Hz}$ sampling rate. Raw fluorescence values of each cell were normalized and analyzed using a custom-made MATLAB script as previously described (Frazzini et al., 2016; Granzotto et al., 2019). The code calculates the number of transients per minute (frequency) and the amplitude of the $\mathrm{Ca}^{2+}$ spikes. Only transients that were $50 \%$ above the baseline were considered.

\section{Neuronal striatal culture immunofluorescence}

Neuronal striatal cultures were grown on $35 \mathrm{~mm}$ glass coverslips. Neurons were washed thoroughly twice in ice-cold PBS, fixed for 10 min at RT with 4\% PFA, permeabilized with PBS $+0.1 \%$ Triton X-100, and then blocked for $1 \mathrm{~h}$ at RT with $1 \%$ of bovine serum albumin in PBS $+0.1 \%$ Tween20 (blocking solution). Cells were incubated for $1 \mathrm{~h}$ at RT with anti-GluN1 antibody (1:200, Alomone) and anti-NOS1 antibody (1:50, Santa Cruz Biotechnology) in the blocking solution. After washing in PBS cultures were stained with species-specific Alexa-conjugated secondary antibodies (Alexa-633, 1:500; Alexa-488, 1:2000, Thermo Fisher Scientific, respectively) for $1.5 \mathrm{~h}$ at room temperature in the dark. Coverslips were then mounted with ProGold-antifade mounting medium (Thermo Fisher Scientific) on cleaned microscopy slides. Cells were imaged on a Zeiss LSM800 confocal microscope equipped with a 63x NA 1.40 Plan-Apochromat oil immersion objective and a super-resolution Airyscan module. Five optical slices (170 nm step size) were acquired for each neuron. After Airyscan processing and background subtraction, images were transformed as maximum orthogonal projections of the whole stack using the ZEN software (Zeiss). Images were further analyzed with the Fiji distribution of ImageJ software as follows. To identify NMDAR-related puncta, GluN1 images were thresholded, the watershed algorithm was applied to define boundaries between the puncta, and finally, binary transformed. The obtained image was used as a mask to measure the number, size, and fluorescent intensity of GluN1 puncta in primarydendrites of nNOS (-) and nNOS (+) neurons.

\section{Assessment of neuronal injury}

Neuronal death was assessed with the lactate dehydrogenase (LDH) efflux assay as previously described (Granzotto and Sensi, 2015). 


\section{Spectroscopic analysis}

To evaluate spectroscopic interferences due to DTNB/DTT application, absorbance spectra of the two compounds were measured. DTNB $(0.5 \mathrm{mM})$ and DTT $(10 \mathrm{mM})$ were dissolved in HCSS, and their absorbance spectra measured at room temperature with a SpectraMax 190 plate reader within the $300-600 \mathrm{~nm}$ range ( $5 \mathrm{~nm}$ step size). Results are reported as optical density (OD).

\section{Statistical analysis}

No statistical methods were employed to determine the sample size. All the results are reported as mean \pm standard error of the mean (SEM). Comparison between two groups was performed with Student's t-test or Welch's corrected unpaired t-test, where appropriate. For comparisons with more than two groups, one-way or two-way ANOVA was performed, where appropriate, followed by Tukey's post-hoc test. Based on conventional criteria, results were considered statistically significant when $p<0.05$. ${ }^{*}$ indicates $p<0.05$ and ${ }^{* *}$ indicates $p<0.01$.

\section{Results}

Spontaneous $\mathrm{Ca}^{2+}$ transients of nNOS (+) neurons are identical to the ones of nNOS (-) neurons

Our neuronal cultures exhibit intracellular $\mathrm{Ca}^{2+}$ transients that depend on synaptic activity and glutamatergic signaling (Frazzini et al., 2016; Granzotto et al., 2019; Isopi et al., 2015). To evaluate potential differences in the activation of excitatory signaling between nNOS $(-)$ and nNOS $(+)$ neurons, we measured spontaneous $\mathrm{Ca}^{2+}$ transients in the two neuronal populations. Striatal neurons were loaded with the high-affinity $\mathrm{Ca}^{2+}$ sensitive dye fluo-4 $\left(\mathrm{K}_{d}=335 \mathrm{nM}\right)$, and changes in $\left[\mathrm{Ca}^{2+}\right]_{i}$ were monitored with microfluorimetry. Changes were analyzed in terms of $\mathrm{Ca}^{2+}$ transient frequency and mean transient amplitude. nNOS (-) and nNOS (+) neurons exhibited overlapping patterns of spontaneous activity as far as spiking frequency (Fig. 1A-C) and transient amplitude (Fig. 1D).

Pharmacological manipulations with tetrodotoxin (TTX, $1 \mu \mathrm{M}$, to block action potentials), and NBQX (2,3-dihydroxy-6-nitro-7-sulfamoyl-benzo[f]quinoxaline, $10 \mu \mathrm{M})$, or dAPV (D-2-amino-5phosphonovaleric acid, $100 \mu \mathrm{M}$ ) to suppress glutamate-mediated effects demonstrated that changes in $\left[\mathrm{Ca}^{2+}\right]_{\mathrm{i}}$ levels are driven by $\mathrm{Ca}^{2+}$ entry resulting from action potential firing and activation of synaptic glutamatergic receptors (Fig. 1E). Experiments performed in a $\mathrm{Ca}^{2+}$-free medium (supplemented with $50 \mu \mathrm{M}$ EDTA) fully abrogated $\left[\mathrm{Ca}^{2+}\right]_{i}$ rises (Fig. 1E), thereby indicating that these resulted from $\mathrm{Ca}^{2+}$ entry and not mobilization of the cation from intraneuronal sites. 

significant differences in terms of spontaneous glutamatergic receptor activation.

Compared to nNOS (-) neurons, nNOS (+) neurons show identical $\mathrm{Ca}^{2+}$ rises following synaptic and extrasynaptic NMDAR activation

Several lines of evidence indicate that synaptic and extrasynaptic NMDARs (synNMDARs and exNMDARs, respectively) exert different effects on neuronal functioning, with exNMDARs playing a key role in the activation of pro-death pathways (Hardingham and Bading, 2010).

To evaluate whether the resilience of nNOS (+) neurons to excitotoxic challenges is driven by differences in exNMDARs, we employed an established pharmacological paradigm that selectively and sequentially activates synNMDARs and exNMDARs. After baseline fluorescence acquisition, fluo4 loaded neurons were exposed to 4-AP (4-aminopyridine, $2.5 \mathrm{mM}$ ), a potassium channel blocker. The maneuver promotes sustained neuronal firing, thereby allowing evaluation of $\mathrm{Ca}^{2+}$ influx through synaptic glutamatergic receptors (i.e., synNMDARs). Neurons were then exposed to MK-801 (10 $\mu \mathrm{M}$, in the presence of 4-AP) to promote complete and irreversible blockade of synNMDARs. Following a brief washout period, neurons were then challenged with NMDA $(50 \mu \mathrm{M})+$ glycine $(10$ $\mu \mathrm{M})$ to allow $\mathrm{Ca}^{2+}$ entry through exNMDARs.

Analysis of the time course of fluo-4 changes, during synNMDAR activation, revealed no differences in terms of $\mathrm{Ca}^{2+}$ rises or cation load between nNOS (-) and nNOS (+) neurons (Fig. 2A-C). Similarly, no significant differences were observed between the two neuronal populations as far as exNMDAR-driven $\left[\mathrm{Ca}^{2+}\right]_{i}$ changes (Fig. 2D-E). Further analysis of $\left[\mathrm{Ca}^{2+}\right]_{i}$ dynamics showed no differences in the cation influx rate (Fig. 2F).

Overall, these findings indicate that nNOS (+) and (-) neurons respond to the activation of synNMDARs and exNMDARs with overlapping changes in $\left[\mathrm{Ca}^{2+}\right]_{i}$, thereby suggesting that the two populations are equipped with similar pools of equally functional synNMDARs and exNMDARs.

Compared to nNOS (-) neurons, nNOS (+) neurons show reduced transcriptomic and protein expression of the NMDAR subunit 1

Previous studies investigating the expression of NMDAR subunits in nNOS (+) neurons have provided contrasting results (Augood et al., 1994; Kim et al., 2001; Landwehrmeyer et al., 1995; Price Jr. et al.,1993; Weiss et al., 1998). To address this question in our system, we performed qRTPCR on a set of selected transcripts obtained from pools of nNOS (-) and nNOS (+) neurons (11 to 18 neurons per sample, Fig. 3A). Analysis of qRT-PCR data showed that, compared to nNOS (-), nNOS (+) 
GluN1 (also known as NR1) (Fig. 3B). Other NMDAR candidate gene transcripts, Grin2a and Grin2b, show no differences between the two populations (Fig. 3B). Expression levels of other genes coding for proteins that have been proposed to be involved in the neuroprotection exhibited by nNOS (+) neurons, like Sod2 and Gpx1, were similar in the two study groups. The $B c / 2$ transcript was not detectable (Fig. 3B and Supplementary Table 1). Of note, Nos1 transcript (encoding the nNOS protein) was found significantly increased in nNOS (+) neuron samples, thereby confirming the selectivity and specificity of our procedure in the isolation of nNOS (+) neurons (Fig. 3B).

Our transcriptomic findings were further validated by interrogating mouse scRNA-Seq data obtained from the Allen Brain Cell Atlas database. We identified two neuronal clusters expressing specific nNOS (+) markers (Nos1+, Sst+, Gad+, Pvalb+, and Npy+; Fig. 3C-D and Supplementary File 1). DEGs assessment was performed by comparing the transcriptome of nNOS (+) neurons with the transcriptome of the broad family of excitatory neurons (Supplementary File 2), a population particularly vulnerable in neurodegenerative settings (Fu et al., 2018). In line with the aim of the study, the downstream analysis focused on elucidating differences in terms of glutamatergic signaling. Supporting our qRT-PCR results, gene ontology (GO) analysis showed that $\mathrm{nNOS}(+)$ neurons display an overall reduction of transcripts associated with excitatory glutamatergic signaling (Fig. 3E and Supplementary File 2). In line with transcriptomic data, GluN1 reduction was confirmed by immunofluorescence (IF) in cultured striatal neurons (Fig. 3F-G).

Compared to $n N O S(-)$, nNOS (+) neurons show reduced NMDAR-driven $\left[\mathrm{Ca}^{2+}\right]_{i}$ rises following receptor reduction

NMDAR ionic conductance can be modulated by oxidizing/reducing agents (Aizenman et al., $1989,1990,2020)$. We evaluated, in nNOS (+) and nNOS (-) neurons, NMDAR activity before and after pharmacological manipulation set to alter the redox status of the receptor (Fig. 4A). After baseline fluorescence acquisition, fura-2 loaded neurons were challenged with NMDA (25 $\mu \mathrm{M})+$

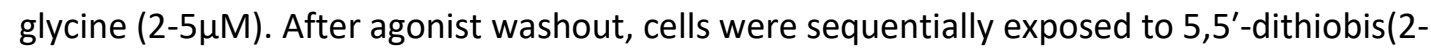
nitrobenzoic acid) (DTNB; $0.5 \mathrm{mM}$ ) and to dithiothreitol (DTT; 2-10 mM), and finally to NMDA (25 $\mu \mathrm{M})+$ glycine $(2-5 \mu \mathrm{M})$. The maneuver allows the evaluation of NMDAR activity in conditions of receptor reduction state (Aizenman et al., 1989). Results were analyzed in fold changes of NMDAdriven $\mathrm{Ca}^{2+}{ }_{\mathrm{i}}$ rises before and after DTNB-DTT exposures. While DTNB-DTT application resulted in a net increase of NMDA-driven $\mathrm{Ca}^{2+}{ }_{i}$ entry in nNOS (-) neurons (Fig. 4B-C), the same maneuver produced significantly lower $\mathrm{Ca}^{2+}{ }_{\mathrm{i}}$ changes in nNOS (+) neurons (Fig. 4B-C). Similar results were observed when cultures were exposed to a higher concentration of DTT (10 mM; Fig. 4C). Of note, DTNB-DTT application did not modify resting $\mathrm{Ca}^{2+}{ }_{\mathrm{i}}$ levels in the two populations (Fig. 4D), thereby 
indicating that fura-2 signals are not affected by artifactual differences in resting levels of cation load.

Overall, this set of experiments supports the notion that nNOS (+) neurons possess a larger pool of fully reduced NMDARs when compared to the general population of nNOS (-) neurons.

Long-term dynamic changes in NMDAR levels could account for nNOS (+) neurons survival following an excitotoxic challenge

nNOS (+) neurons are spared from acute excitotoxic insults by occluding critical steps in the cascade (Canzoniero et al., 2013; Granzotto and Sensi, 2015; Granzotto et al., 2020). To evaluate additional mechanisms of neuroprotection, we set a protocol that mimics long-term excitotoxic insults and imaged functional changes in nNOS (+) neurons.

Serum- and supplement-free Neurobasal medium exchange has been found to produce, in mature, near-pure cultured neurons, widespread neuronal loss, a phenomenon primarily driven by L-cysteine-dependent activation of NMDARs (Hogins et al., 2011; Maggioni et al., 2015; Olney et al., 1990).

In line with a previous report (Hogins et al., 2011), we found that medium exchange resulted in $40 \%$ neuronal loss in our cultures 16 to 24 hours after the challenge (Fig. 5A-B). The toxic effect was abolished when the medium exchange was performed in the presence of dAPV (100 $\mu \mathrm{M})$. Of note, nNOS (+) neurons were largely spared from the maneuver (Fig. 5A, C and unpublished observations).

Thus, we employed the neurobasal medium change maneuver to dissect changes in cells exposed to a long-term excitotoxic environment (Hogins et al., 2011; Olney et al.,1990). Neurobasaltreated cultures were compared with sister cultures challenged in the presence of dAPV. Cultures with no medium exchange (naïve cells) were used as control. Sixteen to twenty hours after the insult cultures were loaded with fura- 2 and NMDA-driven $\mathrm{Ca}^{2+}{ }_{\mathrm{i}}$ changes evaluated. $\mathrm{nNOS}(+)$ and few surviving nNOS (-) neurons did not show alteration in $\left[\mathrm{Ca}^{2+}\right]_{i}$ while dAPV-treated and naïve sister cultures produced significant $\left[\mathrm{Ca}^{2+}\right]_{i}$ elevations (Fig. 5C-G). Of note, naïve and dAPV-treated cultures showed differences in overall cation load (Fig. $5 \mathrm{H}$ ), thereby suggesting that, in our model, dAPV exposures positively affect $\mathrm{Ca}^{2+}$ buffering mechanisms.

To test if the abolished response to NMDA depends on reduced expression or defective functioning of the receptor, we exposed our cultures to a pharmacological maneuver that triggers maximal NMDARs activity. To assess these large $\mathrm{Ca}^{2+}{ }_{i}$ changes, the low-affinity $\mathrm{Ca}^{2+}$ sensor fura-FF $\left(K_{d}=5.5 \mu M\right)$ was used, and cultures were challenged with NMDA $(50 \mu M)+$ glycine $(10 \mu M)$ in a magnesium-free medium supplemented with $10 \mathrm{mM} \mathrm{Ca}^{2+}$. In line with experiments shown in Fig. 5C- 
305

306

307

308

309

310

311

312

313

314

315

316

317

318

319

320

321

322

323

324

325

326

327

328

329

330

331

332

333

334

335

336

337

338

$E$, medium exchange-treated cultures exhibited significantly reduced $\left[\mathrm{Ca}^{2+}\right]_{i}$ amplitudes, overall cation loads, and cation influx rates compared to the control groups (Fig. 5I-L).

Exposures to AMPA or a depolarizing medium (high $\mathrm{K}^{+}$, Fig. $5 \mathrm{M}-\mathrm{Q}$ ) failed to generate $\mathrm{Ca}^{2+}{ }_{\mathrm{i}}$ rises, thereby indicating that $\mathrm{Ca}^{2+}{ }_{i}$ changes were specifically driven by NMDAR activation (Turetsky et al., 1994).

\section{Discussion}

The primary purposes of the study were to 1 ) elucidate the molecular and functional properties of NMDARs in the subpopulation of nNOS (+) neurons and 2) evaluate effects of chronic excitotoxic challenges. Our results integrate our previous findings (Canzoniero et al., 2013; Granzotto and Sensi, 2015) and provide novel insights on the role of NMDARs in nNOS (+) neurons and may help decipher the role of NMDARs under neurodegenerative conditions. Our study supports the notion of dynamic control of NMDAR activity at least in part modulated by the neuronal redox status and the presence of a toxic extracellular milieu.

NMDAR-driven intracellular $\mathrm{Ca}^{2+}$ overload is a mandatory step in the excitotoxic cascade. Compelling evidence, however, suggests that receptor subunit arrangements and localization significantly affect the downstream responses elicited by NMDAR agonists, independently of overall cation accumulation.

The activity of the NMDAR is affected by its subunits (Paoletti et al., 2013). Endogenous modulators like protons, $\mathrm{Zn}^{2+}$, and ROS exert an inhibitory effect (Zhu and Paoletti, 2015) while specific amino acids (glycine and D-serine) or reducing agents potentiate NMDAR activity (Paoletti et al., 2013). The complexity of NMDARs physiology is exacerbated by the divergent action played by synNMDARs and exNMDARs. Evidence indicates that exNMDARs promote cell death signaling in antagonism to synNMDARs that activate anti-apoptotic and neurotrophic pathways (Hardingham et al., 2002; Hardingham and Bading, 2010). We combined functional, transcriptomic, and imaging data to gain further insights into the role of NMDAR activation in the nNOS (+) neurons resilience to excitotoxins.

Our results revealed a composite picture. $\mathrm{Ca}^{2+}$ imaging experiments, in line with our previous reports (Canzoniero et al., 2013; Granzotto and Sensi, 2015), indicate that nNOS (+) neurons possess equally functional pools of NMDARs when compared to nNOS (-) neurons. In addition, no topological differences were observed when evaluating synNMDARs and exNMDARs activity in the two neuronal populations (Fig. 2). Surprisingly, the transcriptomic and histochemical analysis indicated a net decrease in the expression of NMDA GluN1 subunit (Fig. 3) in nNOS (+) neurons, an important 
feature considering that, among the seven different NMDAR subunits, GluN1 plays a mandatory role for the receptor assembling and functioning (Paoletti et al., 2013).

To gain some further insight, we focused on two distinguishing features of the NMDARs and the nNOS (+) subpopulation. NMDARs are modulated by oxidizing and reducing agents, which decrease or potentiate the amplitude of receptor response to agonists, respectively (Aizenman et al.,1989, 1990, 2020). On the other hand, nNOS (+) neurons fail to generate ROS of mitochondrial origin following the activation of NMDARs (Canzoniero et al., 2013; Granzotto and Sensi, 2015). The phenomenon may be related to increased cellular defenses against oxidative/nitrosative damage (Gonzalez-Zulueta et al., 1998; Granzotto and Sensi, 2015; Granzotto et al., 2020). This distinct feature supports the idea that nNOS (+) neurons, by constitutively dealing with an antioxidant milieu, possess fewer but more reduced, and therefore more functional, NMDARs. This notion is supported by findings on NMDAR activity in striatal neurons exposed to DNTB/DTT (Fig. 4). This set of experiments indicates that, upon complete NMDAR reduction, nNOS (+) neurons generate significantly reduced receptor-mediated $\mathrm{Ca}^{2+}{ }_{i}$ amplitudes when compared to nNOS (-) neurons (Figs. 4C). Thus, one can speculate that, in nNOS (+) neurons, a cell-autonomous mechanism regulates GluN1 expression to balance increased receptor functioning. This intriguing hypothesis is supported by complementary evidence showing that non-toxic oxidative challenges, which conceivably reduce NMDAR activity (Aizenman et al., 1990), result in upregulated GluN1 expression (Betzen et al., 2009; Hota et al., 2010; Massaad and Klann, 2011). In addition, this view extends recent findings on the mechanistic liaison between NMDAR activity and the transcription of antioxidant molecules (Baxter et al., 2015; Papadia et al.,2008) and open the possibility for a feedback loop in which the cell redox status may affect the transcription of synaptic proteins and vice versa. This hypothesis may also have implications for disorders in which NMDAR overactivation, increased ROS generation, and alterations in the transcriptional machinery help to modulate neurodegenerative processes.

Moreover, with the limitations of an indirect, population-based approach, our results confirm that $\mathrm{nNOS}(+)$ neurons possess fully operational NMDARs [Figs. 1 and 2 and (Canzoniero et al., 2013; Granzotto and Sensi, 2015)], thereby arguing against the presence of mechanisms of resistance that act upstream in the excitotoxic cascade. We also found that changes in NMDARs may account for nNOS (+) resilience upon chronic excitotoxic hits. This idea is supported by a set of experiments showing that $\mathrm{nNOS}(+)$ neurons fail to respond to NMDA stimulations after prior exposure to chronic excitotoxic challenges (Fig. 5). The effect was found to be specific to NMDARand not AMPAR- or VGCC- dependent activation and mirrors the attenuation of NMDAR activity previously reported in an in vivo model of TBI (Biegon et al., 2004). In this regard, one can speculate 
that nNOS (+) neurons, by missing critical steps of the early stages of the excitotoxic cascade (Canzoniero et al., 2013; Granzotto and Sensi, 2015) activate pathways instrumental for NMDA removal in the attempt to limit the damage associated with chronic excitotoxicity.

Three major evidence supports this idea. First, NMDARs, although reported to be static when compared to AMPARs, possess endocytic motifs that are required for receptor internalization and degradation (Roche et al., 2001; Scott et al., 2004). Second, when stimulated to operate at full capacity, NMDARs generated lower $\left[\mathrm{Ca}^{2+}\right]_{i}$ rises (Figs. $5 \mathrm{I}-\mathrm{L}$ ) when compared to control cultures, thereby suggesting that fewer receptors are present on the cellular surface. The third argument is specific to our experimental setting as the activation of the NMDAR glycine (and L-cysteine) binding site primes the receptor internalization (Nong et al., 2003). In agreement, excitotoxic challenges performed in the absence of NMDAR co-agonists (i.e., glycine or D-serine) produce different functional and viability outcomes (Wu et al., 2017). Of note, this proposed mechanism is not limited to nNOS (+) neurons but can be extended to virtually all those neurons that are spared by our chronic excitotoxic challenge (Fig. 5A-B). However, we cannot exclude the possibility that these subsets of neurons fail to respond to NMDA stimulations for reasons unrelated to receptor expression on the plasma membrane (i.e., negative post-translational modifications, etc.). Another unsolved question is whether the blockade of NMDAR signaling is a regulated process. Further studies aimed at manipulating the underlying mechanism will be required.

\section{Conclusions}

Our findings may have intriguing implications for neurological conditions associated with NMDAR overactivation. The reduced vulnerability of $\mathrm{nNOS}(+)$ neurons indicates that the presence of downstream steps of the cascade can be promising pharmacological targets for neuroprotection (Granzotto et al., 2020). The phenomenon also can limit the side effects associated with the pharmacological blockade of NMDARs (Ikonomidou and Turski, 2002). In addition, the reduced NMDAR responses following chronic excitotoxic hits provide alternative heuristic models to understand the failure of NMDAR antagonists in clinical trials as well as gain insight into the mechanisms associated with the neuroprotective effects exerted by preconditioning against ischemic neuronal death (Aizenman et al., 2000; Dirnagl et al., 2009).

\section{Author contributions}

Conceptualization, Alberto Granzotto and Stefano Sensi; Formal analysis, Alberto Granzotto and Valentina Gatta; Funding acquisition, Alberto Granzotto, Valentina Gatta and Stefano Sensi; Investigation, Alberto Granzotto, Marco d'Aurora and Manuela Bomba; Methodology, Alberto 
407 Granzotto and Valentina Gatta; Project administration, Stefano Sensi; Visualization, Alberto

408 Granzotto; Writing - original draft, Alberto Granzotto; Writing - review \& editing, Alberto Granzotto,

409 Valentina Gatta, Marco Onofrj and Stefano Sensi.

410

411 Acknowledgments

412 The authors thank all the members of the Molecular Neurology Unit for helpful discussions.

413

414 Fundings

415

SLS is supported by research fundings from the Italian Department of Health (RF-2013-

41602358785 and NET-2011-02346784-1), from the AIRAlzh Onlus (ANCC-COOP), from the Alzheimer's

417 Association - Part the Cloud: Translational Research Funding for Alzheimer's Disease (18PTC-19-

418 602325) and theAlzheimer's Association - GAAIN Exploration to Evaluate Novel Alzheimer's Queries

419 (GEENA-Q-19-596282). AG is supported by the European Union's Horizon 2020 research and

420 innovation program under the Marie Skłodowska-Curie grant agreement iMIND - No. 84166. 


\section{References}

Aizenman, E., Hartnett, K. A., and Reynolds, I. J. (1990). Oxygen free radicals regulate NMDAreceptor function via a redox modulatory site. Neuron 5, 841-6. doi:10.1016/0896-6273(90)90343-e.

Aizenman, E., Lipton, S. A., and Loring, R. H. (1989). Selective modulation of NMDA responses byreduction and oxidation. Neuron 2, 1257-63. doi:10.1016/0896-6273(89)90310-3.

Aizenman, E., Loring, R. H., Reynolds, I. J., and Rosenberg, P. A. (2020). The Redox Biology of Excitotoxic Processes: The NMDA Receptor, TOPA Quinone, and the Oxidative Liberation of Intracellular Zinc. Front. Neurosci. 14, 778. doi:10.3389/fnins.2020.00778.

Aizenman, E., Sinor, J. D., Brimecombe, J. C., and Herin, G. A. (2000). Alterations of N-methylD- aspartate receptor properties after chemical ischemia. J. Pharmacol. Exp. Ther. 295, 572-7.Available at: http://www.ncbi.nlm.nih.gov/pubmed/11046090 [Accessed March 30, 2020].

Allen Brain Institute (2019). No Title. Available at: https://portal.brain-map.org/.

Augood, S. J., McGowan, E. M., and Emson, P. C. (1994). Expression of N-methyl-d-aspartate receptor subunit NR1 messenger RNA by identified striatal somatostatin cells. Neuroscience 59,7-12. doi:10.1016/0306-4522(94)90093-0.

Bano, D., Zanetti, F., Mende, Y., and Nicotera, P. (2011). Neurodegenerative processes inHuntington's disease. Cell Death Dis. 2, e228-e228. doi:10.1038/cddis.2011.112.

Baxter, P. S., Bell, K. F. S., Hasel, P., Kaindl, A. M., Fricker, M., Thomson, D., et al. (2015). Synaptic NMDA receptor activity is coupled to the transcriptional control of the glutathione system. Nat. Commun. 6, 6761. doi:10.1038/ncomms7761.

Beal, M. F. (1998). Excitotoxicity and nitric oxide in Parkinson's disease pathogenesis. Ann. Neurol. 44, S110-4. Available at: http://www.ncbi.nlm.nih.gov/pubmed/9749581 [Accessed August 13, 2018].

Betzen, C., White, R., Zehendner, C. M., Pietrowski, E., Bender, B., Luhmann, H. J., et al. (2009). Oxidative stress upregulates the NMDA receptor on cerebrovascular endothelium. Free Radic. Biol. Med. 47, 1212-20. doi:10.1016/j.freeradbiomed.2009.07.034.

Biegon, A., Fry, P.A., Paden, C.M., Alexandrovich, A., Tsenter, J., Shohami, E. (2004) Dynamic changes in N-methyl-d-aspartate receptors after closed head injury in mice: Implications for treatment of neurological and cognitive deficits. Proc. Natl. Acad. Sci. 101, 5117-5122.

Canzoniero, L. M. T., Granzotto, A., Turetsky, D. M., Choi, D. W., Dugan, L. L., and Sensi, S. L. (2013).nNOS(+) striatal neurons, a subpopulation spared in Huntington's Disease, possess 
functional NMDA receptors but fail to generate mitochondrial ROS in response to an excitotoxic challenge. Front. Physiol. 4 MAY, 112. doi:10.3389/fphys.2013.00112.

Choi, D. W. (1988). Calcium-mediated neurotoxicity: relationship to specific channel types and role inischemic damage. Trends Neurosci. 11, 465-469. doi:10.1016/0166-2236(88)90200-7.

Choi, D. W. (1992). Excitotoxic cell death. J. Neurobiol. 23, 1261-1276. doi:10.1002/neu.480230915.Choi, D. W. (2020). Excitotoxicity: Still Hammering the Ischemic Brain in 2020. Front. Neurosci. 14, 1104. doi:10.3389/FNINS.2020.579953.

Csernansky, C. A., Canzoniero, L. M. T., Sensi, S. L., Yu, S. P., and Choi, D. W. (1994). Delayed application of aurintricarboxylic acid reduces glutamate-induced cortical neuronal injury. J. Neurosci. Res. 38, 101-108. doi:10.1002/JNR.490380113.

Dawson, T. M., Bredt, D. S., Fotuhi, M., Hwang, P. M., and Snyder, S. H. (1991). Nitric oxide synthaseand neuronal NADPH diaphorase are identical in brain and peripheral tissues. Proc. Natl. Acad.Sci. U. S. A. 88, 7797-7801.

Dirnagl, U., Becker, K., and Meisel, A. (2009). Preconditioning and tolerance against cerebral ischaemia: from experimental strategies to clinical use. Lancet. Neurol. 8, 398-412.

Ferrante, R. J., Kowall, N. W., Beal, M. F., Richardson Jr., E. P., Bird, E. D., and Martin, J. B. (1985). Selective sparing of a class of striatal neurons in Huntington's disease. Science (80-. ). 230, 561563.

Frazzini, V., Guarnieri, S., Bomba, M., Navarra, R., Morabito, C., Mariggiò, M. A. A., et al. (2016). Altered Kv2.1 functioning promotes increased excitability in hippocampal neurons of an Alzheimer's disease mouse model. Cell Death Dis. 7, e2100. doi:10.1038/cddis.2016.18.

Gonzalez-Zulueta, M., Ensz, L. M., Mukhina, G., Lebovitz, R. M., Zwacka, R. M., Engelhardt, J. F., et al. (1998). Manganese superoxide dismutase protects nNOS neurons from NMDA and nitric oxide-mediated neurotoxicity. J. Neurosci. 18, 2040-55.

Granzotto, A., Bomba, M., Castelli, V., Navarra, R., Massetti, N., D’Aurora, M., et al. (2019). Inhibitionof de novo ceramide biosynthesis affects aging phenotype in an in vitro model of neuronal senescence. Aging (Albany. NY). 11, 6336-6357. doi:10.18632/aging.102191.

Granzotto, A., Canzoniero, L. M. T., and Sensi, S. L. (2020). A Neurotoxic Ménage-à-trois: Glutamate,Calcium, and Zinc in the Excitotoxic Cascade. Front. Mol. Neurosci. 13, 225. doi:10.3389/fnmol.2020.600089.

Granzotto, A., and Sensi, S. L. (2015). Intracellular zinc is a critical intermediate in the excitotoxiccascade. Neurobiol. Dis. 81, 25-37. doi:10.1016/j.nbd.2015.04.010. 
Graveland, G. A., Williams, R. S., and Difiglia, M. (1985). Evidence for degenerative and regenerativechanges in neostriatal spiny neurons in Huntington's disease. Science 227, 770-3.

Grynkiewicz, G., Poenie, M., and Tsien, R. Y. (1985). A new generation of Ca2+ indicators with greatlyimproved fluorescence properties. J. Biol. Chem. 260, 3440-3450.

Hardingham, G. E., and Bading, H. (2010). Synaptic versus extrasynaptic NMDA receptor signalling:implications for neurodegenerative disorders. Nat. Rev. Neurosci. 11, 682-96. doi:10.1038/nrn2911.

Hardingham, G. E., Fukunaga, Y., and Bading, H. (2002). Extrasynaptic NMDARs oppose synapticNMDARs by triggering CREB shut-off and cell death pathways. Nat. Neurosci. 5, 405-14. doi:10.1038/nn835.

Hogins, J., Crawford, D. C., Zorumski, C. F., and Mennerick, S. (2011). Excitotoxicity triggered byNeurobasal culture medium. PLoS One 6, e25633. doi:10.1371/journal.pone.0025633.

Hope, B. T., Michael, G. J., Knigge, K. M., and Vincent, S. R. (1991). Neuronal NADPH diaphorase is anitric oxide synthase. Proc. Natl. Acad. Sci. U. S. A. 88, 2811-2814.

Hota, S. K., Hota, K. B., Prasad, D., Ilavazhagan, G., and Singh, S. B. (2010). Oxidative-stressinduced alterations in Sp factors mediate transcriptional regulation of the NR1 subunit in hippocampusduring hypoxia. Free Radic. Biol. Med. 49, 178-91. doi:10.1016/j.freeradbiomed.2010.03.027.

Hynd, M. R., Scott, H. L., and Dodd, P. R. (2004). Glutamate-mediated excitotoxicity andneurodegeneration in Alzheimer's disease. Neurochem. Int. 45, 583-595. doi:10.1016/j.neuint.2004.03.007.

Ikonomidou, C., and Turski, L. (2002). Why did NMDA receptor antagonists fail clinical trials for stroke and traumatic brain injury? Lancet. Neurol. 1, 383-6. doi:10.1016/s14744422(02)00164-3.

Isopi, E., Granzotto, A., Corona, C., Bomba, M., Ciavardelli, D., Curcio, M., et al. (2015). Pyruvate prevents the development of age-dependent cognitive deficits in a mouse model of Alzheimer's disease without reducing amyloid and tau pathology. Neurobiol. Dis. 81, 214-224. doi:10.1016/j.nbd.2014.11.013.

Kim, D. Y., Kim, S. H., Choi, H. B., Min, C., and Gwag, B. J. (2001). High Abundance of GluR1 mRNA and Reduced Q/R Editing of GluR2 mRNA in Individual NADPH-Diaphorase Neurons. Mol. Cell. Neurosci. 17, 1025-1033. doi:10.1006/MCNE.2001.0988.

Koh, J. Y., and Choi, D. W. (1988). Vulnerability of cultured cortical neurons to damage by excitotoxins: differential susceptibility of neurons containing NADPH-diaphorase. J. Neurosci. 8, 2153-2163. 
Koh, J. Y., Peters, S., and Choi, D. W. (1986). Neurons containing NADPH-diaphorase are selectivelyresistant to quinolinate toxicity. Science (80-. ). 234, 73-76.

Landwehrmeyer, G. B., Standaert, D. G., Testa, C. M., Penney Jr., J. B., and Young, A. B. (1995). NMDA receptor subunit mRNA expression by projection neurons and interneurons in rat striatum. J. Neurosci. 15, 5297-5307.

Lau, A., and Tymianski, M. (2010). Glutamate receptors, neurotoxicity and neurodegeneration. Pflügers Arch. Eur. J. Physiol. 460, 525-542. doi:10.1007/s00424-010-0809-1.

Lein, E. S., Hawrylycz, M. J., Ao, N., Ayres, M., Bensinger, A., Bernard, A., et al. (2007). Genomewideatlas of gene expression in the adult mouse brain. Nature 445, 168-176. doi:10.1038/nature05453.

Lewerenz, J., and Maher, P. (2015). Chronic Glutamate Toxicity in Neurodegenerative DiseasesWhat is the Evidence? Front. Neurosci. 9, 469. doi:10.3389/fnins.2015.00469.

Maggioni, D., Monfrini, M., Ravasi, M., Tredici, G., and Scuteri, A. (2015). Neurobasal mediumtoxicity on mature cortical neurons. Neuroreport 26, 320-324. doi:10.1097/WNR.0000000000000343.

Massaad, C. A., and Klann, E. (2011). Reactive oxygen species in the regulation of synaptic plasticityand memory. Antioxid. Redox Signal. 14, 2013-54. doi:10.1089/ars.2010.3208.

Mufson, E. J., and Brandabur, M. M. (1994). Sparing of NADPH-diaphorase striatal neurons inParkinson's and Alzheimer's diseases. Neuroreport 5, 705-8.

Nong, Y., Huang, Y.-Q., Ju, W., Kalia, L. V., Ahmadian, G., Wang, Y. T., et al. (2003). Glycine bindingprimes NMDA receptor internalization. Nature 422, 302-307. doi:10.1038/nature01497.

Olney, J. W., Zorumski, C., Price, M. T., and Labruyere, J. (1990). L-cysteine, a bicarbonatesensitiveendogenous excitotoxin. Science 248, 596-9. doi:10.1126/science.2185543.

Paoletti, P., Bellone, C., and Zhou, Q. (2013). NMDA receptor subunit diversity: impact on receptorproperties, synaptic plasticity and disease. Nat. Rev. Neurosci. 14, 383-400. doi:10.1038/nrn3504.

Papadia, S., Soriano, F. X., Léveillé, F., Martel, M.-A., Dakin, K. A., Hansen, H. H., et al. (2008). Synaptic NMDA receptor activity boosts intrinsic antioxidant defenses. Nat. Neurosci. 11,476-87. doi:10.1038/nn2071.

Price Jr., R. H., Mayer, B., and Beitz, A. J. (1993). Nitric oxide synthase neurons in rat brain express more NMDA receptor mRNA than non-NOS neurons. Neuroreport 4, 807-810. 
555

556

557

558

559

560

561

562

563

564

565

566

567

568

569

570

571

572

573

574

575

576

577

578

579

580

581

582

583

584

585

586

587

588

Roche, K. W., Standley, S., McCallum, J., Dune Ly, C., Ehlers, M. D., and Wenthold, R. J. (2001). Molecular determinants of NMDA receptor internalization. Nat. Neurosci. 4, 794-802. doi:10.1038/90498.

Scott, D. B., Michailidis, I., Mu, Y., Logothetis, D., and Ehlers, M. D. (2004). Endocytosis and degradative sorting of NMDA receptors by conserved membrane-proximal signals. J. Neurosci. 24, 7096-109. doi:10.1523/JNEUROSCI.0780-04.2004.

Sensi, S. L., Paoletti, P., Bush, A. I., and Sekler, I. (2009). Zinc in the physiology and pathology of theCNS. Nat. Rev. Neurosci. 10, 780-91. doi:10.1038/nrn2734.

Tasic, B., Yao, Z., Graybuck, L. T., Smith, K. A., Nguyen, T. N., Bertagnolli, D., et al. (2018). Shared anddistinct transcriptomic cell types across neocortical areas. Nature 563, 72-78. doi:10.1038/s41586-018-0654-5.

Turetsky, D. M., Canzoniero, L. M. T., Sensi, S. L., Weiss, J. H., Goldberg, M. P., and Choi, D. W. (1994). Cortical neurones exhibiting kainate-activated co2+ uptake are selectively vulnerable to ampa/kainate receptor-mediated toxicity. Neurobiol. Dis. 1, 101-110. doi:10.1006/nbdi.1994.0013.

Uemura, Y., Kowall, N. W., and Beal, M. F. (1990). Selective sparing of NADPH-diaphorasesomatostatin-neuropeptide $Y$ neurons in ischemic gerbil striatum. Ann. Neurol. 27, 620625.doi:10.1002/ana.410270606.

Wang, J., and Swanson, R. A. (2020). Superoxide and Non-ionotropic Signaling in Neuronal Excitotoxicity. Front. Neurosci. 4, 861. doi:10.3389/fnins.2020.00861.

Weiss, J. H., Turetsky, D., Wilke, G., and Choi, D. W. (1994). AMPA/kainate receptor-mediated damage to NADPH-diaphorase-containing neurons is Ca2+ dependent. Neurosci. Lett. 167,93-6.

Weiss, S. W., Albers, D. S., ladarola, M. J., Dawson, T. M., Dawson, V. L., and Standaert, D. G. (1998). NMDAR1 glutamate receptor subunit isoforms in neostriatal, neocortical, and hippocampalnitric oxide synthase neurons. J. Neurosci. 18, 1725-34. doi:10.1523/JNEUROSCI.18-05-01725.1998.

Wu, Y., Chen, C., Yang, Q., Jiao, M., and Qiu, S. (2017). Endocytosis of GluN2B-containing NMDAreceptors mediates NMDA-induced excitotoxicity. Mol. Pain 13, 1744806917701921. doi:10.1177/1744806917701921.

Zhu, S., and Paoletti, P. (2015). Allosteric modulators of NMDA receptors: multiple sites and mechanisms. Curr. Opin. Pharmacol. 20, 14-23. doi:10.1016/j.coph.2014.10.009. Zivin, J. A., and Choi, D. W. (1991). Stroke Therapy. Sci. Am. 265, 56 63.doi:10.1038/scientificamerican0791-56. 
A
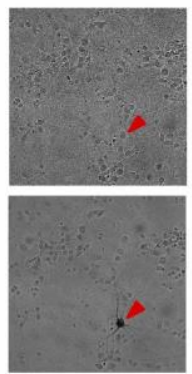

Low
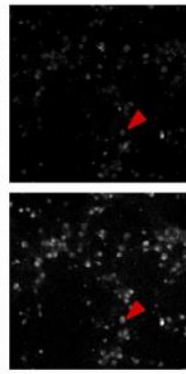

$\left[\mathrm{Ca}^{2+}\right]_{1}$
C

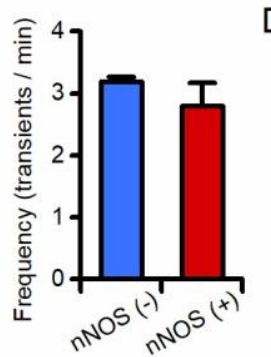

D

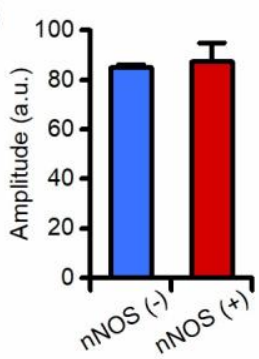

B
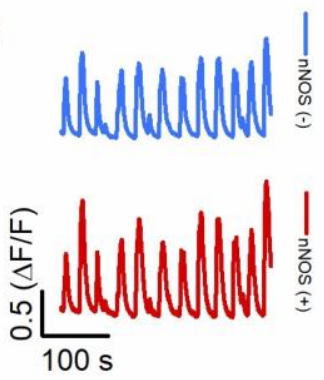

E $+\mathrm{dAPV}$
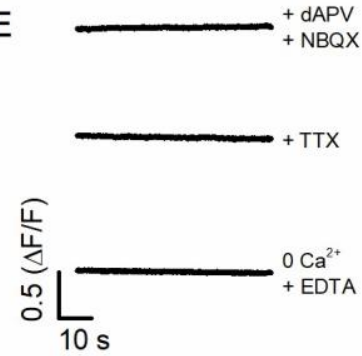

590

591

592

593

594

595

596

597

598

599

600

601

602

603

604

605

Figure 1. $\mathrm{nNOS}(+)$ and the general population of $\mathrm{nNOS}(-)$ neurons show overlapping spontaneous

$\mathrm{Ca}^{2+}$ transients. (A) Striatal neurons were loaded with fluo-4 to monitor spontaneous $\mathrm{Ca}^{2+}$ transients in vitro. Left panels show phase contrast images of the assayed field before (top) and after (bottom) NADPH-diaphorase staining (cell with dark precipitate, red arrowhead); right panels show greyscalecolored images of fluo-4 loaded cultures before (top) and during (bottom) a $\mathrm{Ca}^{2+}$ transient. Images are representative of 7 independent experiments. (B) Representative time courses of $\mathrm{Ca}^{2+}$ transients occurring in nNOS (-) and nNOS (+) neurons. (C) Bar graph depicts $\mathrm{Ca}^{2+}$ transients frequency values obtained in the two populations (transients/min in nNOS (-): $3.19 \pm 0.07$ vs. $2.8 \pm 0.37$ in nNOS (+), p $=0.50, n=616$ nNOS (-) vs. $n=10$ nNOS (+) neurons from 7 independent experiments). (D) Bar graph depicts $\mathrm{Ca}^{2+}$ transients amplitude values obtained from the same groups as in C (fluo-4 peak amplitude in nNOS (-): $85.01 \pm 1.29$ vs. $87.39 \pm 7.55$ in nNOS (+) neurons, $p=0.81$ ). (E)

Representative time courses of $\mathrm{Ca}^{2+}$ transients occurring in striatal neurons treated with glutamate receptors antagonists (dAPV and NBQX, $100 \mu \mathrm{M}$ and $10 \mu \mathrm{M}$, respectively), $T \mathrm{TX}(1 \mu \mathrm{M})$, or a $\mathrm{Ca}^{2+}$-free medium. 
A
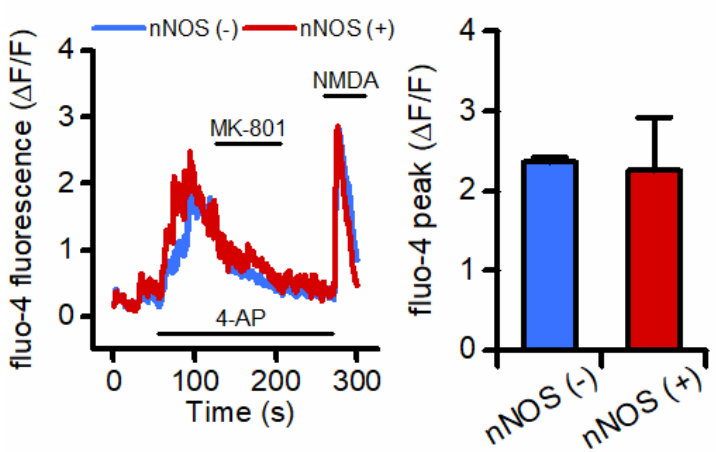

D

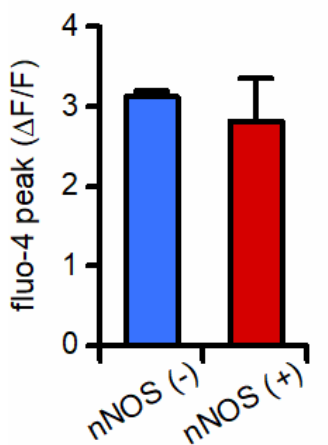

B
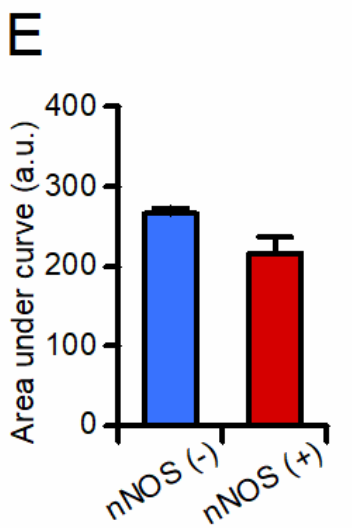

C

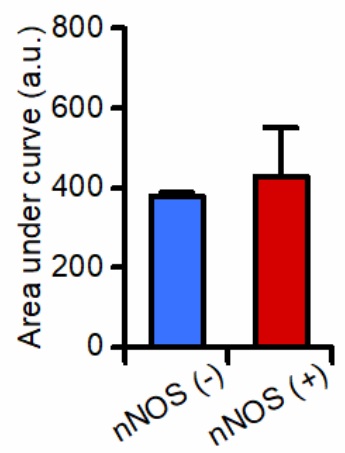

$\mathrm{F}$

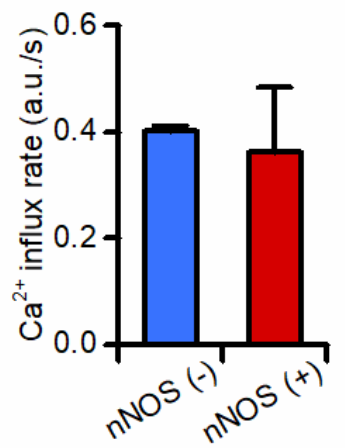

607

608

Figure 2. $\mathrm{nNOS}(+)$ and the general population of $\mathrm{nNOS}(-)$ neurons show overlapping intracellular $\mathrm{Ca}^{2+}$ rises upon activation of synaptic and extrasynaptic NMDARs. (A) Representative time courses of fluo-4 loaded nNOS (-) and nNOS (+) striatal neurons exposed to a pharmacological maneuver set to activate synNMDARS and exNMDARs. (B) Bar graph depicts peak of $\left[\mathrm{Ca}^{2+}\right]_{i}$ values obtained in the two populations during synNMDAR activation [fluo-4 peak in nNOS (-): $2.36 \pm 0.05$ vs. $2.25 \pm 0.66$ in nNOS (+) neurons, $\mathrm{p}=0.81, \mathrm{n}=389 \mathrm{nNOS}(-)$ vs. $\mathrm{n}=5 \mathrm{nNOS}(+)$ neurons from 4 independent experiments]. (C) Bar graph depicts cumulative $\left[\mathrm{Ca}^{2+}\right]_{i}$ changes in the two populations expressed as area under the curve (AUC) of arbitrary units (a.u.) during synNMDAR activation [fluo-4 AUC in nNOS $(-): 379.28 \pm 8.84$ vs. $429.01 \pm 122.89$ in nNOS (+) neurons, $p=0.53$ ]

(D) Bar graph depicts peak of $\left[\mathrm{Ca}^{2+}\right]_{i}$ values obtained in the two populations during exNMDAR activation [fluo-4 peak in nNOS (-): $3.12 \pm 0.06$ vs. $2.81 \pm 0.53$ in nNOS (+) neurons, $p=0.59$ ]. (E) Bar graph depicts cumulative $\left[\mathrm{Ca}^{2+}\right]_{i}$ changes in the two populations during exNMDAR activation [fluo-4 AUC in nNOS (-): $266.08 \pm 7.33 \mathrm{vs}$. populations expressed as a.u. changes per second during exNMDAR activation (influx rate in nNOS () $0.40 \pm 0.009$ vs. $0.36 \pm 0.12$ in nNOS (+) neurons, $p=0.63$ ] 
A

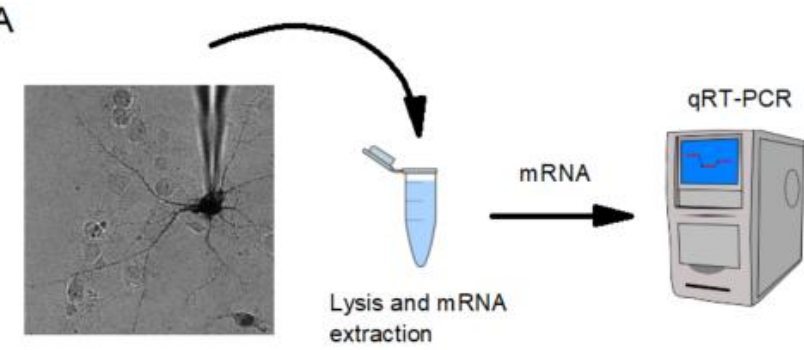

B

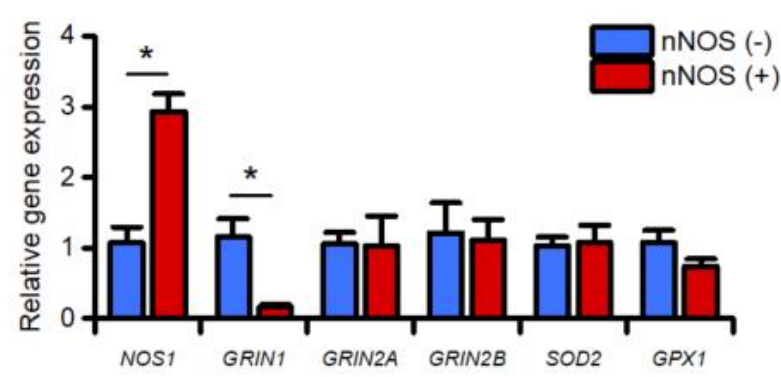

$\mathrm{F}$
nNOS (-)

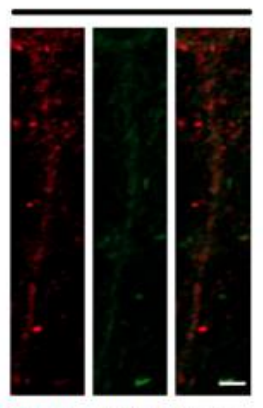

GluN1 NOS1 Merge
G nNOS (+)

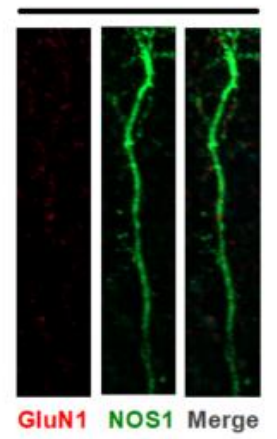

626
C

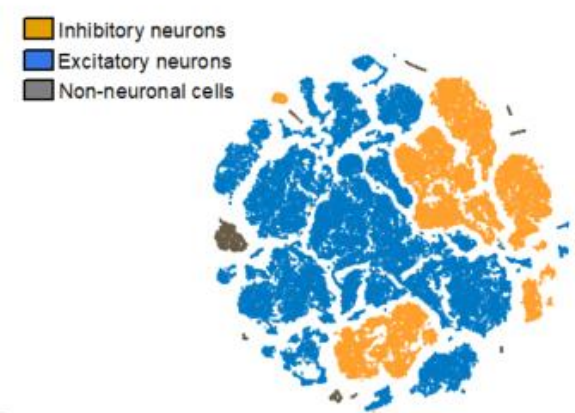

D

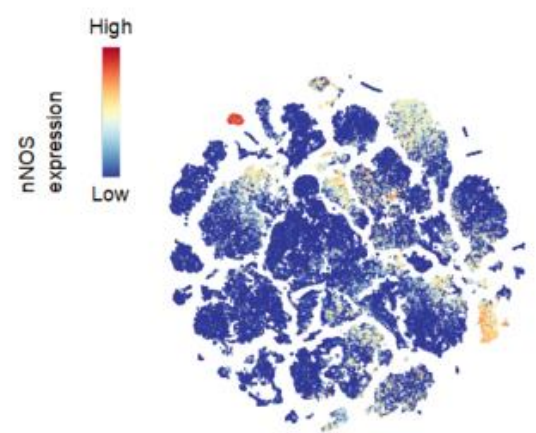

E

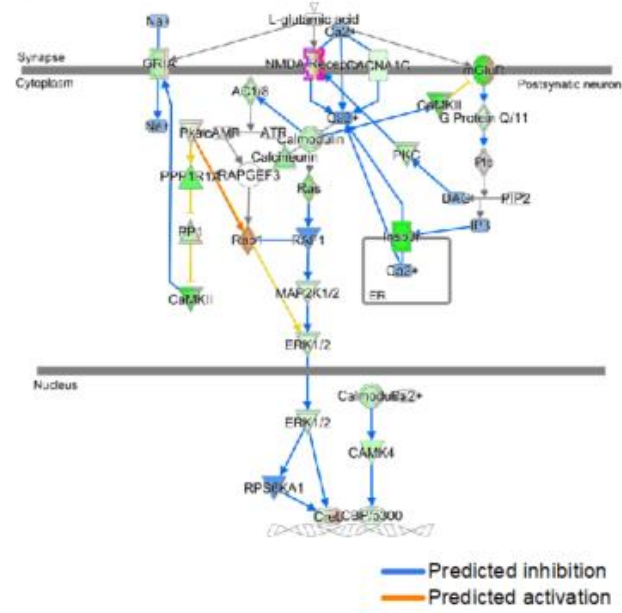

Figure 3. nNOS (+) neurons show a decreased expression of the NMDA receptor subunit GluN1.

(A) The pictogram illustrates the "patch-like" procedure employed to isolate mRNA obtained from single nNOS (+) neurons. The same approach was employed to isolate nNOS (-) neurons employed as control. (B) Bar graphs illustrate changes in mRNA levels of the indicated genes measured by realtime PCR in nNOS (+) neurons when compared to the general population of nNOS (-) cells (relative Nos1 expression in nNOS (-): $1.07 \pm 0.22$ vs. $2.92 \pm 0.25$ in nNOS (+) neurons, $\mathrm{p}<0.001$; relative Grin 1 expression in nNOS (-): $1.15 \pm 0.25$ vs. $0.16 \pm 0.02$ in nNOS (+) neurons, $p=0.005 ; n=4-5$ replicates). No differences were observed in the other tested transcripts (see Supplementary Table 1). (C) t-SNE plot of the scRNA-Seq database from the Allen Brain Atlas. Clusters are color-coded basedon cell classification as inhibitory neurons (yellow), excitatory neurons (blue), or non-neuronal cells (gray). 
637 (D) t-SNE plot of the same dataset as in C showing levels of nNOS expression. Please, note the

638 presence of a high nNOS expressing cluster (top left) identified as bona fide nNOS (+) neurons. (E)

639 The pictogram illustrates the glutamatergic signaling Pathway Activity Analysis. The analysis, based

640 on the expression of significantly perturbed genes from our dataset, predicts if the pathway is

641 activated (yellow-orange arrows) or inhibited (blue arrows) in nNOS (+) neurons when compared to

642 excitatory neurons. Please, note the consistent inhibition of glutamatergic signaling. (F)

643 Representative super-resolution confocal images of dendrites obtained from striatal nNOS (-) (left

644 panel) and nNOS (+) neurons and stained with anti-GluN1 (red) and anti-NOS1 (green) antibodies

645 (scale bar $=2 \mu \mathrm{m}$ ). (G) Bar graph depicts quantification of dendritic GluN1-related fluorescent

646 intensity [normalized GluN1 signal in nNOS (-): $1.00 \pm 0.04$ vs. $0.66 \pm 0.05$ in nNOS (+) neurons,

$647 p<0.001, n=43-44$ dendrites from at least 3 independent experiments]. 
A

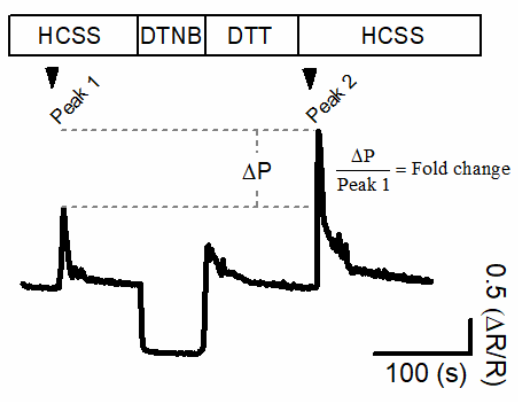

C

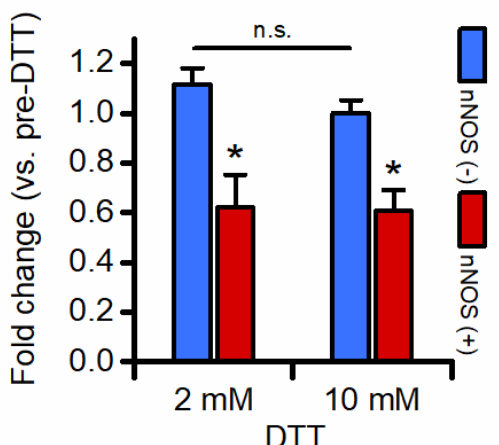

B

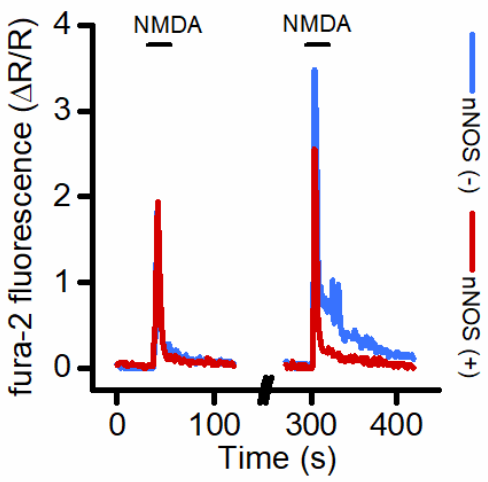

$\mathrm{D}$

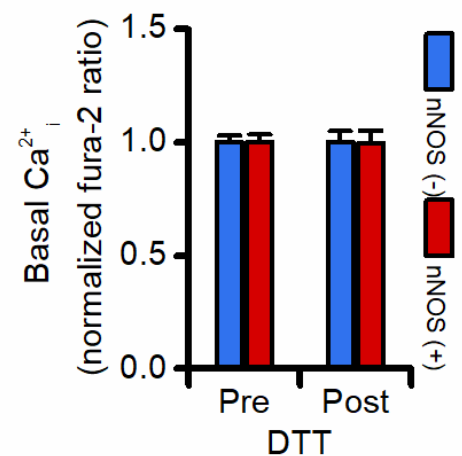

pharmacological receptor reduction. (A) The pictogram illustrates the pharmacological protocol set to evaluate agonist-dependent changes in $\left[\mathrm{Ca}^{2+}\right]_{i}$ rises before and after NMDAR oxidation/reduction. Please, note that the sharp decrease in fura-2 signal upon DTNB exposure is due to spectroscopic interferences between the probe and the drug (Supplementary fig. 1). (B) Representative time courses of fura-2 loaded nNOS (-) and nNOS (+) striatal neurons exposed to NMDA before (left traces) and after (right traces) full receptor reduction (for clarity, traces during DTNB and DTT exposure were omitted). (C) Bar graphs depict quantification of experiments in B expressed as fold changes in $\left[\mathrm{Ca}^{2+}\right]_{\mathrm{i}}$ rises following exposure to $2 \mathrm{mM}$ (left panel) or $10 \mathrm{mM}$ (right panel) DTT in the two populations (DTT $2 \mathrm{mM}$, fura-2 fold change in nNOS: (-) 1 (a)

(-) $1.11 \pm 0.06$ vs. $0.61 \pm 0.13$ in $\mathrm{nNOS}(+)$ neurons, $p=0.005, n=389$ nNOS (-) vs. $n=9$ nNOS (+) neurons from 7 independent experiments; 10 $\mathrm{mM}$, fura-2 fold change in nNOS (-): $0.99 \pm 0.05$ vs. $0.60 \pm 0.08$ in nNOS (+) neurons, $p=0.002, n=$ 342 nNOS (-) vs. $\mathrm{n}=7 \mathrm{nNOS}(+)$ neurons from 5 independent experiments). (D) Bar graph depicts basal $\mathrm{Ca}^{2+}{ }_{\mathrm{i}}$ levels in nNOS (-) and nNOS (+) neurons before and after DTT exposure expressed as normalized fura-2 ratio. n.s. $=$ not significant. 

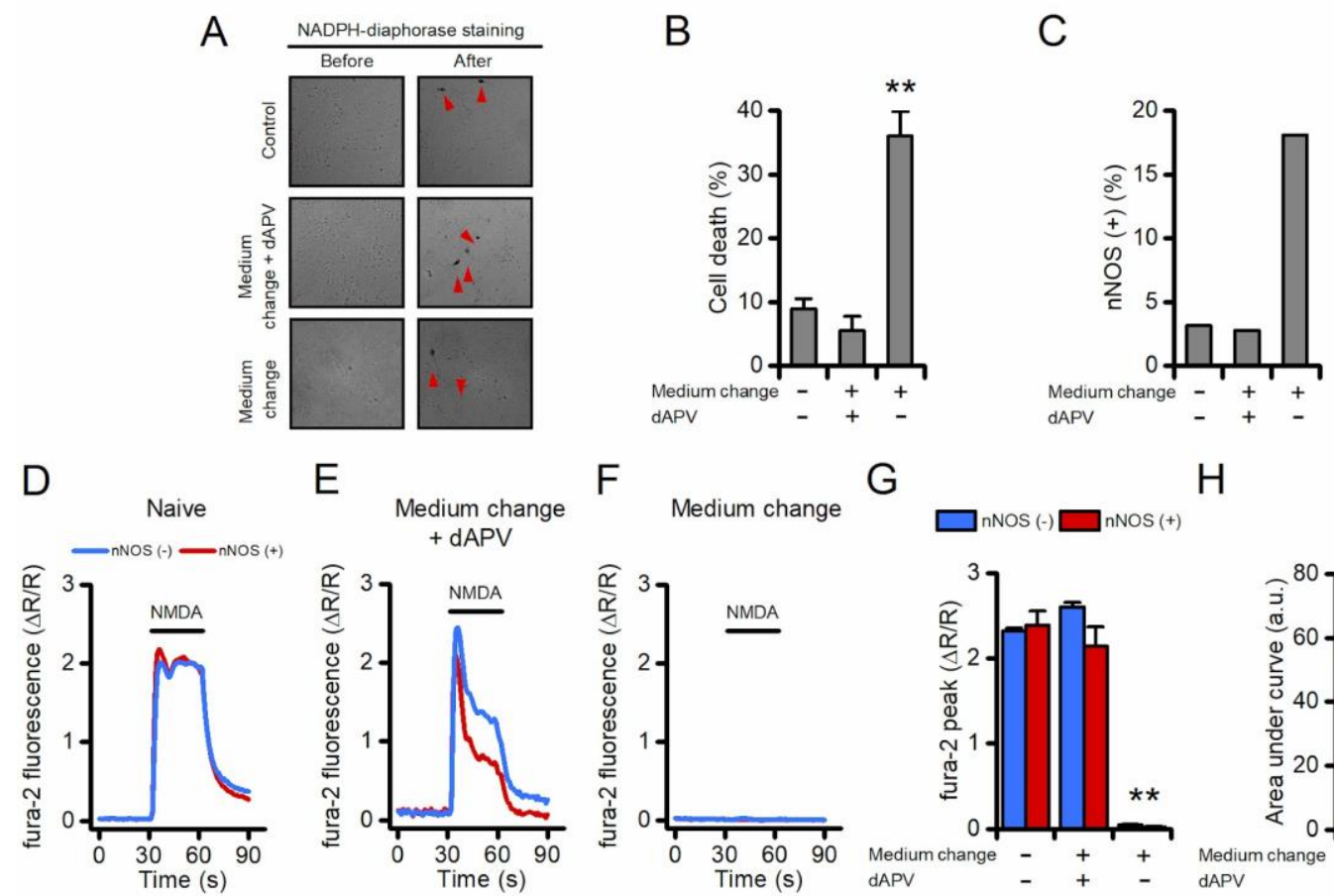

$\mathrm{H}$
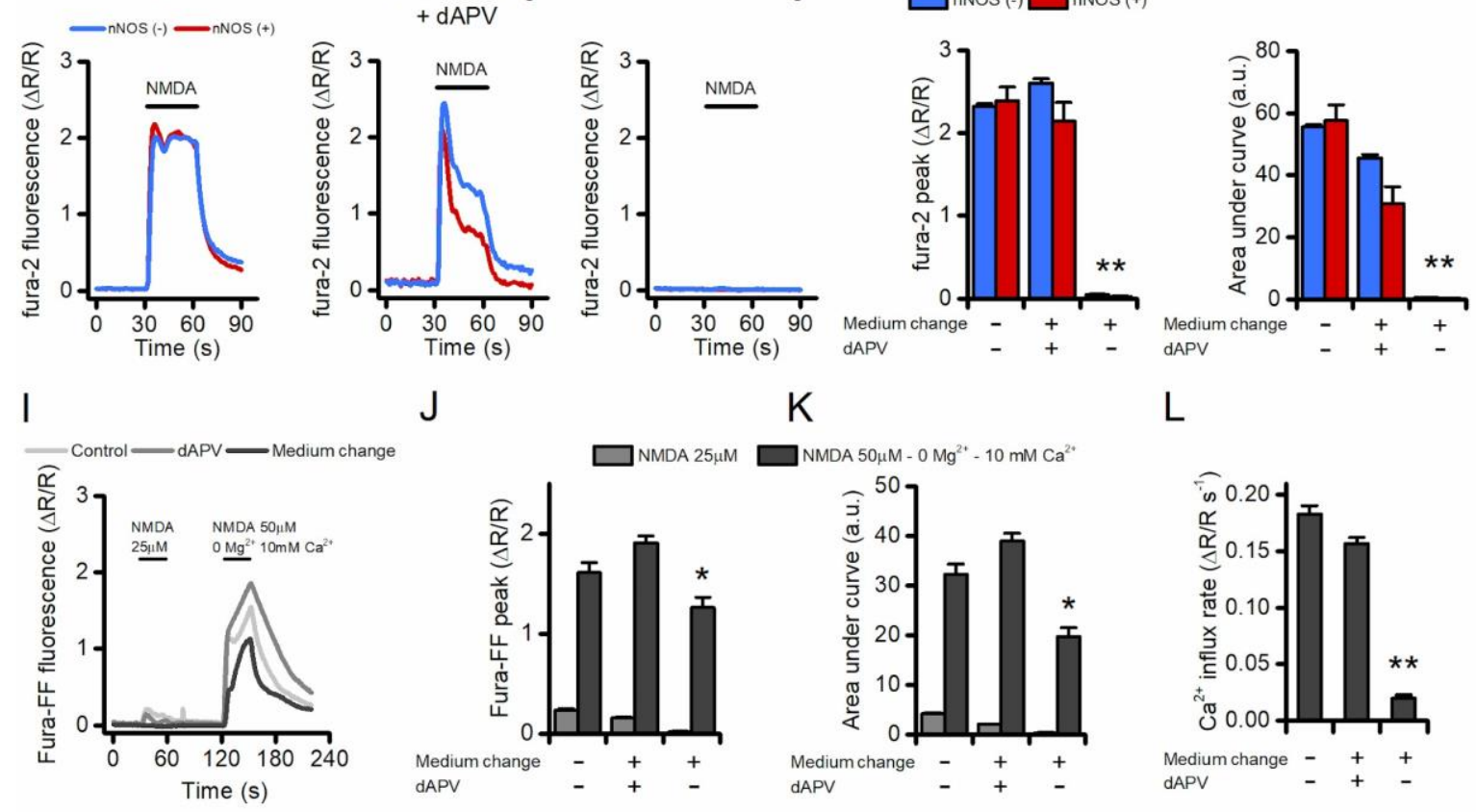

K

$\mathrm{L}$
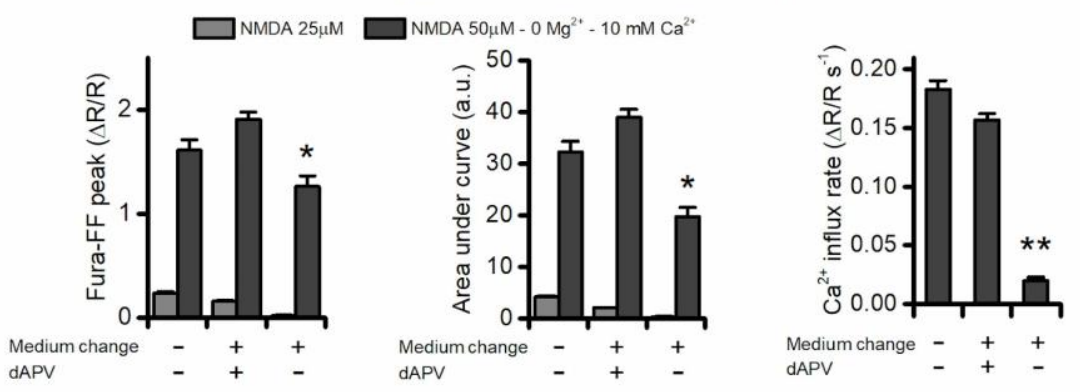

$\mathrm{M}$

$\mathrm{N}$

$\mathrm{O}$

$\mathrm{P}$

Q

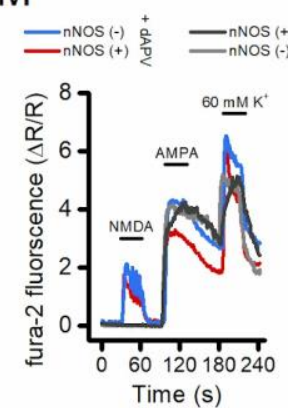

Time (s)

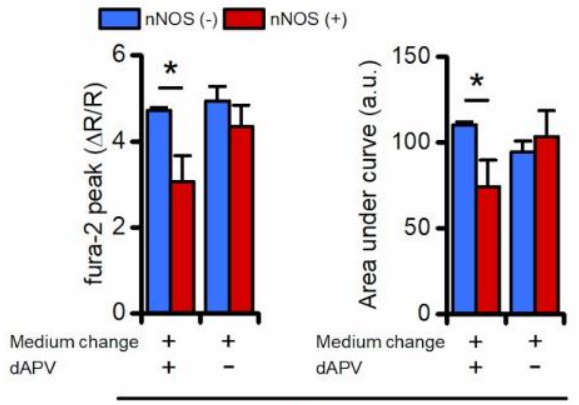

AMPARs

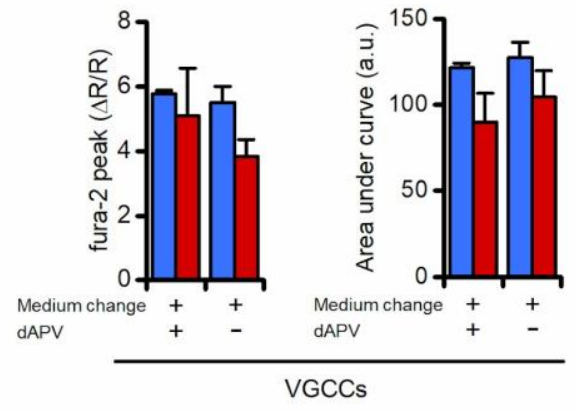

Figure 5. Neurons spared after an excitotoxic challenge fail to respond to NMDA stimulation.

670 (A) The pictogram illustrates phase contrast images of untreated (top), medium change + dAPV-

671 treated (middle), and medium change-treated (bottom) neuronal striatal cultures before (left) and

672 after (right) the NADPH-diaphorase staining. Red arrowheads indicate nNOS (+) neurons. (B) Bar graph depicts the vulnerability of striatal cultures exposed to the treatments described in A. 
674

675

676

677

678

679

680

681

682

683

684

685

686

687

688

689

690

691

692

693

694

695

696

697

698

699

700

701

702

703

704

Neuronal viability was assessed, with LDH efflux assay, $16 \mathrm{~h}$ after the challenge (neuronal death in naïve neurons: $8.9 \pm 1.5 \%$ vs. $5.4 \pm 2.2 \%$ in dAPV group vs. $36.0 \pm 3.8 \%$ in medium exchange group, $F(2,45)=37.56, p<0.0001)$. (C) Bar graph depicts the increased number of nNOS (+) neurons, expressed as \% of the live neurons, following medium exchange treatment which is indicative of a relative sparing of the subpopulation. (D-F) Representative time courses of fura-2 loaded nNOS (-) and nNOS (+) striatal neurons exposed to NMDA (25 $\mu \mathrm{M}+2-5 \mu \mathrm{M}$ glycine) 16-20 $\mathrm{h}$ after being exposed to the indicated treatment. (G) Bar graphs show quantification of fura-2 peak values obtained from experiments shown in D-F (treatment effect $F_{(2,1510)}=119.7, p<0.0001$; cell type effect $F_{(2,1510)}=0.9163, p=0.33$; interaction $\left.F_{(2,1510)}=1.357, p=0.25\right)$. $(H)$ Bar graphs show quantification of cumulative $\left[\mathrm{Ca}^{2+}\right]_{i}$ changes obtained from experiments shown in D-F (treatment effect $F_{(2,1510)}=107.7, p<0.0001$; cell type effect $F_{(2,1510)}=1.793, p=0.18$; interaction $F_{(2,1510)}=$ $2.690, p=0.07)$. (I) Representative time courses of fura-FF loaded neuronal striatal cultures exposed to $25 \mu \mathrm{M}$ NMDA (2-5 $\mu \mathrm{M}$ glycine) or $50 \mu \mathrm{M}$ NMDA (10 $\mu \mathrm{M}$ glycine) in a $\mathrm{Mg}^{2+}$-free medium supplemented with $10 \mathrm{mM} \mathrm{CaCl}_{2}$ and assessed $16-20 \mathrm{~h}$ after being challenged with the indicated treatment. (J) Bar graph shows quantification of fura-FF peak values obtained from experiments shown in $\mathrm{I}\left(\mathrm{F}_{(2,402)}=16.11, \mathrm{p}<0.0001\right)$. (K) Bar graphs show quantification of cumulative $\left[\mathrm{Ca}^{2+}\right]_{\mathrm{i}}$ changes obtained from experimentsshown in $\mathrm{I}\left(\mathrm{F}_{(2,402)}=33.43, \mathrm{p}<0.0001\right)$. (L) Bar graph depicts $\mathrm{Ca}^{2+}$ influx rate in the three treatment groups expressed as a.u. changes per second during the first $5 \mathrm{~s}$ of the $50 \mu \mathrm{M}$ NMDA stimulation $\left(\mathrm{F}_{(2,402)}=209.2, \mathrm{p}<0.0001\right)$. (M) Representative time courses of fura-2 loaded nNOS (-) and nNOS (+) striatal neurons sequentially exposed to NMDA ( $25 \mu \mathrm{M}+2-5 \mu \mathrm{M}$ glycine), AMPA (100 $\mu \mathrm{M}+$ cyclothiazide), or a high $\mathrm{K}^{+}$solution (60 $\mathrm{mM} \mathrm{K}^{+}, 10 \mu \mathrm{M} \mathrm{MK}-801,10 \mu \mathrm{M}$ NBQX). (N) Bar graph shows quantificationof fura-2 peak values obtained from neurons exposed to AMPA (treatment effect $F_{(1,459)}=2.889, p=0.09$; cell type effect $F_{(1,459)}=6.383, p=0.01$; interaction $\left.F_{(1,459)}=1.382, p=0.24\right)$. (O) Bar graph shows quantification of cumulative $\left[\mathrm{Ca}^{2+}\right]_{i}$ changes obtained from neurons exposed to AMPA (treatment effect $F_{(1,459)}=0.4273, p=0.51$; cell type effect $F_{(1,459)}=$ 1.622, $p=0.20$; interaction $\left.F_{(1,459)}=4.577, p=0.03\right)$. $(P)$ Bar graph shows quantification of fura-2 peak values obtained from neurons exposed to ahigh $K^{+}$solution (treatment effect $F_{(1,459)}=1.137, p$ $=0.28$; cell type effect $F_{(1,459)}=2.676, p=0.10$; interaction $\left.F_{(1,459)}=0.4707, p=0.49\right)$. (Q) Bar graph shows quantification of cumulative $\left[\mathrm{Ca}^{2+}\right]_{i}$ changes obtained from neurons exposed to a high $\mathrm{K}^{+}$ solution (treatment effect $F_{(1,459)}=0.4063, p=0.52$; cell type effect $F_{(1,459)}=2.760, p=0.09$; interaction $\left.\mathrm{F}_{(1,459)}=0.073, \mathrm{p}=0.78\right)$. 


\section{Supplementary table 1}

\begin{tabular}{|c|c|c|c|}
\hline Gene & nNOS (-) & nNOS (+) & $\boldsymbol{P}$ \\
\hline Nos1 & $1.07 \pm 0.22$ & $2.92 \pm 0.25$ & 0.0003 \\
\hline Grin1 & $1.15 \pm 0.25$ & $0.16 \pm 0.02$ & 0.0272 \\
\hline Grin2a & $1.05 \pm 0.16$ & $1.02 \pm 0.42$ & 0.31 \\
\hline Grin2b & $1.21 \pm 0.42$ & $1.11 \pm 0.29$ & 0.42 \\
\hline Sod2 & $1.03 \pm 0.12$ & $1.07 \pm 0.24$ & 0.42 \\
\hline Gpx1 & $1.06 \pm 0.17$ & $0.73 \pm 0.1$ & 0.08 \\
\hline Bcl2 & ND & ND & \\
\hline
\end{tabular}


A

B

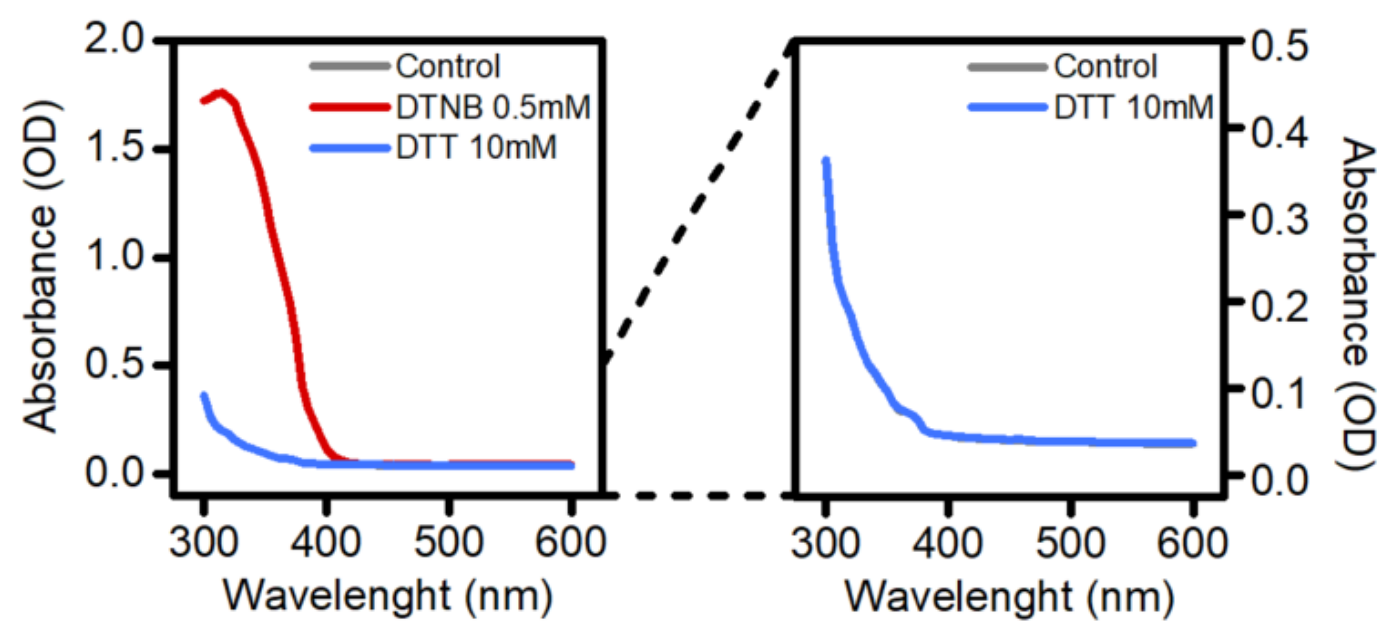

Supplementary figure 1. DTNB interferes with fura-2 excitation wavelengths. (A) The pictogram illustrates DTNB $(0.5 \mathrm{mM})$ and DTT (10 mM) absorption spectra recorded in the $300-600 \mathrm{~nm}$ range ( $5 \mathrm{~nm}$ step size). Please note that DTNB shows maximum absorbance at $340 \mathrm{~nm}$ thereby interfering with the short fura-2 excitation wavelength $(340 / 380 \mathrm{~nm})$. (B) The pictogram shows a magnification of DTT spectrum. Please, note the complete overlap between DTT and control medium traces. 